\title{
Considering sustainability in the planning of transit systems: Istanbul case
}

\author{
B. Buran ${ }^{1}$ \& O. Feyzioğlu ${ }^{2}$ \\ ${ }^{1}$ Transportation Planning Department, IETT, Turkey \\ ${ }^{2}$ Department of Industrial Engineering, Galatasaray University, Turkey
}

\begin{abstract}
Traditionally, minimization of the total travel time spent by the passenger in the system is the most preferred objective while planning transit systems. To satisfy this objective, forecasted travel demands between zones and characteristics of the available fleet are taken into account, and at the end of the planning process, optimum frequencies of each transit lines are identified. Unfortunately, this planning approach is not sufficient as the negative effects of the global warming are growing each day. Fossil fuels are the primary energy sources for transport systems and accordingly, the emission of the greenhouse gases especially carbon dioxide is accredited to this industry. Hence, it is impossible to ignore environmental requirements in the transit planning phase. In this study, we developed a bi-level and bi-objective optimization model to identify the optimum line frequencies. One of the objectives is to minimize the mean travel time of the passengers. The other objective is to minimize the total emission. A genetic algorithm is developed to solve this mathematical programming problem. A large instance related to Istanbul transit system involving 39 zones and 463 bus lines is solved with this solution method and results are elaborated.
\end{abstract}

Keywords: sustainable transit planning, greenhouse gases emission, bi-level programming, genetic algorithm.

\section{Introduction}

Public transportation planning covers a very wide research area. From the design of networks to the rostering of crews, from the evaluation of demand to the transit trip assignment, from mathematical methods of resolution to evolutionary ones, the process of generating a public transportation system has been 
approached from many sides. This thorough interest is partly due to the fact that the development of public transportation is a crucial topic in the modern society. From the users' perspective, the system should meet the demand by providing cheap and direct service to passengers. From the operator's perspective on the opposite, the objective is for the system to make as much profit as possible. It is the main challenge in transit planning to find equilibrium between these conflicting objectives [1].

Due to the traffic congestion, passengers total travel times are increased. Time is a nonrenewable resource so travel and waiting times should be minimized by public transportation system. Another aspect related to the transportation is that it is a major source of pollution and greenhouse gases (GHS) especially carbon dioxide $\left(\mathrm{CO}_{2}\right)$. Greenhouse gas emissions of public transportation are increasing at a faster rate than any other energy using sector. According to International Association of Public Transport (UITP) survey, 23\% percent of total $\mathrm{CO}_{2}$ gases are composed by transportation; including rail, bus, sea, air transportation systems and 98 of all land transport depends on fossil fuels [2].

Governments produce new policies to provide sustainability of transportation systems. There are different solutions for sustainability transport. Advantages in technology renewable sources are used for transportation with costly investments such as using solar, wind or biofuel. The other way of providing sustainability transport is to optimize transit line frequencies regard with minimizing greenhouse gas emissions.

Sustainable transport implies finding a proper balance between (current and future) environmental, social and economic qualities [3]. Environmental, social and economic qualities have different effect on the passengers. Their content can vary with passenger perspective. Sustainable transportation should provide these qualities. For the environmental side different transport modes are responsible for approximately $30 \%$ of global warming. This ratio is much larger compared to those of energy production or industry. In Europe, even despite increasingly cleaner engines, $\mathrm{CO}_{2}$ emissions have not decreased, but keep growing $(+25 \%$ since 1990) [4]. Global warming has become one of the critical issues all over the world. Governments and organizations focus on this topic.

The paper is organized is as follows: we present a brief literature of previous work done related with our model in the first section. Then, a bi-level line frequencies optimization model that has the objective to reduce average traveler transit time and $\mathrm{CO}_{2}$ emissions is presented. As the proposed model is bi-level, a special solution algorithm is developed and its details are given in section four. To show the usefulness and efficiency of our approach, a case study of Istanbul bus network which is one of the largest bus network in the world, is investigated and the results are elaborated. The final section contains some concluding remarks and future research directions.

\section{Literature survey}

In the literature, the public transit planning process is usually divided in a sequence of five steps: (1) the design of routes, (2) the setting of frequencies, 
(3) the timetabling, (4) the vehicle scheduling and (5) the crew scheduling and rostering [1].

\subsection{Transit assignment}

Transit assignment models concerns the selection of routes (paths) between origins and destinations in transportation network. These model objectives are minimizing the total expected travel and waiting time [5-7] perceived total travel times [8], expected travel cost [9, 10], general cost [11] (including; in-vehicle time, waiting time, walking time, a line change penalty), total vehicle operation cost [12], optimize transit lines timetables with fleet size and vehicle services [13], utility maximization [14]. Transit assignment models are solved with the gradient projection method, minimum cost hyperpath search algorithm, method of successive averages algorithm, iterated local search algorithm and Newton method.

\subsection{Stochastic transit assignment}

Stochastic transit assignment models present more accurate results than deterministic ones. When conditions are not stable (perceived travel time, perceived waiting time, etc.), the stochastic transit assignment models are used to ensure more realistic models. Objectives of stochastic transit assignment model can be minimizing generalized cost; in-vehicle time; waiting time; walking time; and a time penalty for each line change with timetable and route choice variables. This model is solved branch and bound algorithm and its application is The Hong Kong Mass Transit Railway [15]. Minimizing perceived cost stochastic transit assignment model with route choice, day to-day variability, OD demand with varying different times, frequency variables is solved method of successive averages algorithm [16].

\subsection{Bi-level programming}

The bi-level programming problem is a special case of multilevel programming problems with a two level structure. There exist many problems in transportation that can be formulated as a bi-level programming problem. Bi-level programming models for the transportation systems are transit frequency, market equilibrium, measurement of gas emissions throughout a traffic network, transit frequency design models. Upper level objective functions can be related to toll revenue, investments, time, accessibility, and so on. The lower level objective functions can be minimizing the travel costs, minimizing travel time. These types of models are solved with TAPAS algorithm, gradient projection method and special improved algorithms [17-19].

\subsection{Sustainable transportation}

In the literature, there are lots of studies for providing sustainable transportation. While some of them are related to analyzing recent negative results for our 
world, the others propose a model to improve transportation system. Soria Alves et al. [20] present a strategic planning model for vehicular air pollution analysis. The model is based on the representation of the urban, transportation and environment conditions interacting under a neural gee-spatial approach to forecast vehicular air pollution for the stage of diagnosis, as a contribution for an urban and transportation sustainability, A case study in Nagoya City Area was conducted to verify the efficiency of the model and to evaluate the best NN structure and also changes in the hidden and output layers were simulated, The result reached expresses an efficient definition of the NN.

To quantify total emissions and compare with the emissions from the additional traffic, Simões et al. [21] propose a study of the environmental impact of urban buses in a real inner city situation. The study is focused on several routes of CARRIS, the company responsible for exploring the bus routes within the central area of Lisbon, Portugal. Based on the urban buses fleet of CARRIS, the topography of selected routes, the vehicles average speed and transported passengers, the CORINAIR methodology (that was supported by European Environment Agency's Topic Centre on Air Emissions) is used to predict fuel consumption and CO, NOX, VOC and particles emissions from these urban buses. Wadhwa [22] proposes a study which has several approaches to achieving transportation sustainability are identified in it. These are grouped into technological, economic and behavioural, and planning and management approaches. Although there are definite viable and feasible options in each approach, their implementation requires strong political will and some not very popular decisions.

Latini et al. [23] presents an overview on air-pollution problems in a complex valley, located in the middle of Italy, and then discusses current and ongoing new deals for reducing transport-related air pollution. This incorporates both "how to control transport" and "how to improve air quality. The dispersion of road-traffic emissions has been modelled using the Gaussian Dispersion Model AERMOD-PRIME. AERMOD is a steady-state plume-dispersion model for assessment of pollutant concentrations from a variety of sources. For this assessment, AERMOD-PRIME has been used to calculate concentrations of CO $\left(\mathrm{mg} / \mathrm{m}^{3}\right)$ under prevailing weather conditions.

In this paper, we propose bi-objective bi-level optimization model to determine transit line frequencies. The two objectives considered at the upper level of our model are minimization of mean passenger travel time and total $\mathrm{CO}_{2}$ emission. At the lower level, we aim to reduce the total passenger travel times given passenger demand and existing lines. The overall model is solved by an adapted genetic algorithm. With these unique features, our work fills an important gap in the literature.

\section{Transit assignment model}

Let us denote $G(\mathcal{N}, \mathcal{A})$ the directed graph with $\mathcal{N}$ as the set of nodes and $A$ is the set of links. Let also $\mathcal{S}$ denotes the set of destination nodes, $\mathcal{A}_{i}^{+}$and $\mathcal{A}_{i}^{-}$the forward and backward star of node $i$ respectively, and $\mathcal{L}$ the set of transit lines. 


\subsection{Upper level problem}

When transit lines frequencies are optimized, the aim of transportation planning authority is to minimize the total travel time spent by the travellers in the network under the fleet size and line constraints. This traditional objective is also considered in our study. The other objective is to minimize the total $\mathrm{CO}_{2}$ emission due to the operating vehicles. When transit line frequencies are increased, the total travel time decreases. Meanwhile, increasing bus frequencies also leads to an increase in the total emission. In other words, these two objectives are in conflict. When our bi-objective model in eqns. (1)-(4) is solved, a set of Pareto optimum or non-dominated solutions are generated. Then, the planning authority has the opportunity to choose the time-emission pair that best suits its purpose.

$$
\begin{gathered}
\operatorname{minimize} \frac{1}{D} \sum_{s \in \mathcal{S}}\left(\sum_{a \in \mathcal{A}} t_{a} v_{a}^{s}+\sum_{i \in N} w_{i}^{s}\right) \\
\operatorname{minimize} \sum_{l \in \mathcal{L}} e_{l} f_{l} \\
\text { s.t. } \sum_{l \in \mathcal{L}} t_{l} f_{l} \leq M \\
f_{l} \geq \underline{f}_{l} \quad l \in \mathcal{L}
\end{gathered}
$$

Here, $D$ denotes the total travel demand between zones, $t_{a}$ the fixed travel time on link $a, v_{a}^{s}$ the flow on link $a$ with destination $s, w_{i}^{s}$ the total waiting time for passengers on node $i$ with destination $s, e_{l}$ the total $\mathrm{CO}_{2}$ emission for a vehicle operating on line $l$, and $t_{l}$ the fixed in-vehicle travel time of line $l, M$ the fleet size, and $\underline{f}_{l}$ the required minimum frequency for line $l$. The variable of this model is $f_{l}$ which denotes the vehicle frequency for line $l$. In eqn. (1), we aim to minimize mean passenger travel time, while in eqn. (2) we aim to minimize the total network emission. Constraint eqn. (3) limits the total number of vehicles to be operated and constraint eqn. (4) lower bounds the frequency of a line.

\subsection{Lower level problem}

$$
\begin{gathered}
\operatorname{minimize} \sum_{s \in \mathcal{S}}\left(\sum_{a \in \mathcal{A}} t_{a} v_{a}^{s}+\sum_{i \in N} w_{i}^{s}\right) \\
\text { s.t. } \sum_{a \in \mathcal{A}_{i}^{+}} v_{a}^{s}-\sum_{a \in \mathcal{A}_{i}^{-}} v_{a}^{s}=g_{i}^{s} \\
v_{a}^{s} \leq\left(\sum_{l \in \mathcal{L}} \delta_{a l} f_{l}\right) w_{i}^{s} a \in \mathcal{A}_{i}^{+}, i \in \mathcal{N}, s \in \mathcal{S}
\end{gathered}
$$




$$
v_{a}^{s} \geq 0 \quad a \in \mathcal{A}, s \in \mathcal{S}
$$

Here $g_{i}^{s}$ is the passenger demand at node $i$ willing to reach destination $s$. The objective in eqn. (5) is to reduce the total passenger travel times. Constraint in eqn. (6) is the general flow balance constraint for network flows: the number of passengers leaving node $i$ must be equal to the sum of the passenger incoming to and waiting at node $i$. Assuming that the passengers waiting at a node could take the first vehicle, constraint eqn. (7) relates links flows and nodes waiting times. Finally constraint eqn. (8) is for non-negative flows.

\section{Solution method}

\subsection{Solving lower level problem}

The proposed model in eqns. (5)-(8) is a large linear programming model. As the optimum solution of the lower level model will be needed several times when the upper level model will be solved iteratively, the lower level model should be solved efficiently. The first thing that can be remarked about the model in eqns. (5)-(8) is that it can be decomposed based on the destination. Hence, the overall problem can be solved optimally if each of the destination decomposed subproblem is solved optimally. If the special structure of the destination based decomposition is investigated, its similarity to the shortest-path problem can be recognized. In fact, shortest-path problem is a special case of our destination based decomposition $\left(f_{l} \rightarrow \infty\right.$ and $w_{i} \rightarrow 0$ ). The algorithm provided below efficiently solves the sub-problem related to destination node $q$ [24].

\section{Transit Route Choice Algorithm:}

1. $u_{i}=\infty$ for all $i \in \mathcal{N} /\{q\}, u_{q}=0, \bar{f}_{i}=0$ for all $i \in \mathcal{N}$, $\mathcal{B}=\mathcal{A}, \overline{\mathcal{A}}=\emptyset$.

2. If $\mathcal{B}=\emptyset$ then go step 3

Otherwise

Find $a=(i, j)$ such that $u_{j}+t_{a}$ is the smallest value of $\mathcal{B}$

$$
\mathcal{B}=\mathcal{B} /\{a\}
$$

If $u_{i} \geq u_{j}+t_{a}$ then

$$
\begin{aligned}
& u_{i}=\left(\bar{f}_{i} u_{i}+f_{a}\left(u_{j}+t_{a}\right)\right) /\left(\bar{f}_{i}+f_{a}\right) \\
& \bar{f}_{i}=\bar{f}_{i}+f_{a} \\
& \overline{\mathcal{A}}=\overline{\mathcal{A}} \cup\{a\}
\end{aligned}
$$

Go back step 2

3. $V_{i}=g_{i}^{q}$ for all $i \in \mathcal{N}$

4. For every link $a \in \overline{\mathcal{A}}$ in decreasing order of $\left(u_{j}+t_{a}\right)$ do

$$
\begin{aligned}
& v_{a}^{q}=\left(f_{a} / \bar{f}_{i}\right) V_{i} \\
& V_{j}=V_{j}+v_{a}^{q}
\end{aligned}
$$

For all others arcs $a \in \mathcal{A} / \overline{\mathcal{A}}$ set $v_{a}^{q}=0$. 


\section{5. $w_{i}^{q}=V_{i} / \bar{f}_{i}$ for all $i \in \mathcal{N}$}

\subsection{Solving overall problem}

We make use of the Non-Dominated Sorting Genetic Algorithm (NSGA-II) [25] to solve the bi-level and bi-objective model given in eqns. (1)-(8). Within the general framework of genetic algorithm, every individual is represented with a vector of size $|\mathcal{L}|$ of real numbers. In other words, our objective is to identify what must be the frequency for each transit line. The steps of our adapted algorithm are given below:

1. Create the initial population by randomly selecting line frequencies that satisfy constraints eqn. (3) and eqn. (4) for each individual that made the population.

2. Find objective function values for the existing population. For each individual, first calculate the objective in eqn. (2). Then given line frequencies, use the transit route choice algorithm to find optimum line flows and waiting times. With this solution, the objective in eqn. (1) can be calculated.

3. Based on the objective functions values, calculate the non-dominance ranking and crowding distance of each individual.

4. To form the new population, first conduct a tournament among individuals to form the mating pool. The tournament is played by two or more individuals that are selected from the existing population and the one that has the lowest non-dominance ranking is added to the mating pool. If there are two or more players that have the lowest ranking score, the one with the largest crowding distance is added to the pool. If there is a tie, the individual to be added to the pool is selected at random. The tournament process continues until the mating pool is filled.

5. Form the new population with the crossover and mutation of the individuals at the mating pool. Two parents are selected from the mating pool, and two children are created with their crossover. Then mutation occurs with a given probability. While parents return to the mating pool, the children are added to the new population. This process continues until the new population reaches to a determined size. Here it is ensured that constraints eqn. (3) and eqn. (4) are satisfied while genetic operators are applied.

6. If the maximum number of iterations is not reached, then go to step 2. Otherwise, identify non-dominated solutions from the existing population and display as a result.

\section{Case study: Istanbul bus network}

To demonstrate the efficiency of our approach, we applied it to İstanbul bus network. İstanbul bus network is very large with 39 zones connected through 590 bus lines. As some lines are used very infrequently, we only investigated 463 of them. The total number of trips is 26,000 during all day. Although there are private companies operating buses in İstanbul, the central bus and bus rapid transit (BRT) network planning and management authority is Istanbul 
Electricity, Tramway and Tunnel (IETT). The total fleet size is around 5000 vehicles (IETT + privately owned). The fleet is composed of very different vehicles. However, we did not take this fact into account directly to keep our model simple. Instead, we assumed that the network is served with an average vehicle. The CO2 emission of this average vehicle is set to $0.850 \mathrm{~kg} / \mathrm{km}$ (Federal Test Procedure normalized) [26].

In this study, the morning peak (07:00AM-09:00AM) transit demand estimates for year 2011 are considered. Around 30\% of the total transit demand is served bus lines.

The estimated daily number of passengers for bus system is 3.5 million and around $30 \%$ of this demand is served during peak morning hours. The data are obtained from the Department of Transportation Planning of İstanbul Metropolitan Municipality.

NSGA-II is run for a population size 100, tournament size 2, crossover rate 0.80 , Pareto front population fraction 0.20 . As it can be observed from fig. 1 , the population average of objective function values start to stabilize around 120 iterations. Hence the maximum number of iterations for the NSGA-II is set to 120. For the crossover operator, we first create a random binary vector.

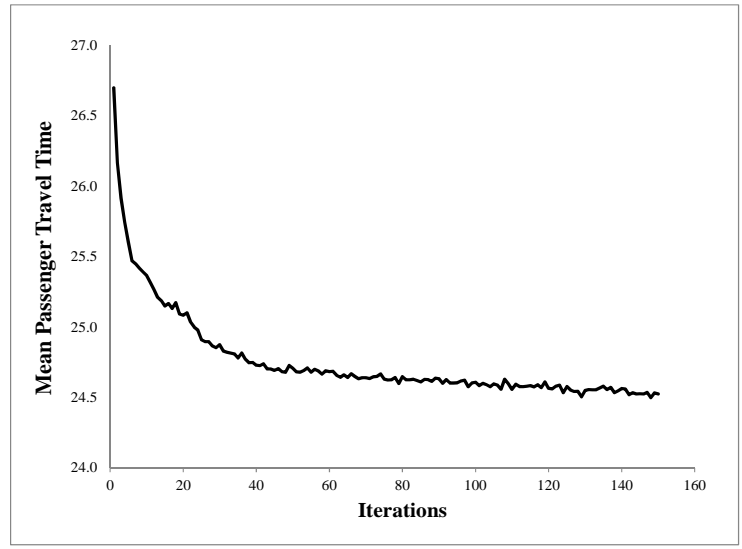

Figure 1: Plot of the population average of mean user time objective during the iterations of NSGA-II.

We then select the genes where the vector is a 1 from the first parent, and the genes where the vector is a 0 from the second parent, and combines the genes to form the child. For the mutation operator, a small number of solution vector elements are selected at random and the values of these elements are randomly increased or decreased. Both operators are arranged such that the produced children are always feasible. As we do not want to discontinue any existing line, the lower bound on the minimum frequency of each line is set to one.

Final results for 5 different runs of the NSGA-II are pooled and the final Pareto front is obtained after removing dominated solutions from this pool. These solutions are shown in fig. 1 . The current frequency assignment is also 
shown in that figure. This solution is dominated by the Pareto optimum solutions of our algorithm. While it is possible to reduce mean passenger travel time for the same $\mathrm{CO}_{2}$ level around $15 \%$, it is interesting to observe that there is a room to cut more than a half of the total $\mathrm{CO}_{2}$ emission for the same mean travel time level. This result is not difficult to explain because most of this type of transit networks are designed to minimize the total or mean passenger travel time. Moreover, many lines of the network are not operated harshly on the efficiency principle. Instead, many lines are continued despite low ridership or long travel distances.

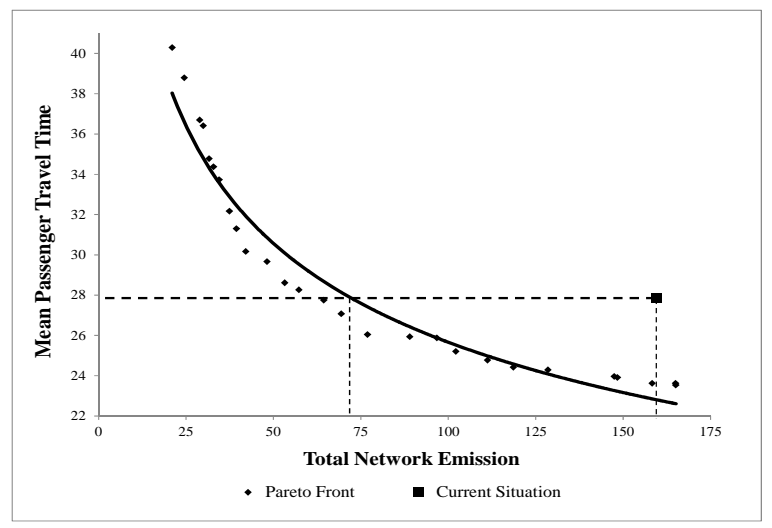

Figure 2: $\quad$ Pareto optimal solutions depicted in the objective functions space.

The Pareto optimal solutions are provided but which one of them is sustainable is not fully answered. Surely solutions lying on the two extreme of the trend curve in fig. 2 are not sustainable: they ignore the passengers in favor of environment or vice versa. We can ignore them. However, identifying the sustainable solution is not simple. In our case, if we had a specific figure of what is the sustainable in terms of per capita $\mathrm{CO}_{2}$ emission and per capita transport time, we could then detect easily which of the Pareto optimal solutions is sustainable or how much these solutions are far from the sustainable solution. As these numbers are non-existing (in fact there is no a common understanding on these numbers), we should make an assumption and accept the solution that is "good enough" in both objectives as the most appropriate. Here we adopt the following convention: the solution that is "good enough" in both objectives is the one with minimal distance to the origin at the objective functions space. As the origin in this space corresponds to the ideal solution (yet impossible to attain), the closest Pareto optimal solution to the ideal solution can be considered as satisfactory. The distance is measured with Euclidean norm and paying equal importance (equal weights) to both upper level objectives. In our case, the solution which results in 28.61 minutes for the mean passenger travel time and 53.24 tonnes of $\mathrm{CO}_{2}$ emission is a good solution. Compared to the current 
situation, the adaptation of this solution may lead to a slight increase (3\%) in mean travel time but also to a significant emission reduction (66\%).

\section{Conclusion and future research}

While frequencies of transit systems lines are planned, minimization of the total travel time spent by the passengers is the most preferred objective. Unfortunately, this planning approach is not sufficient today. Fossil fuels are the primary energy sources for transport systems and accordingly, the emission of greenhouse gases especially carbon dioxide is accredited to transportation industry. Hence, it is impossible to ignore environmental requirements in the transit planning phase. In this study, we develop a bi-level and bi-objective optimization model to identify the optimum line frequencies. One of the objectives is to minimize the mean travel time of the passengers while the other objective is to minimize the total emission. The efficiency of the model is demonstrated with a case study on Istanbul bus network.

This study has the potential of being a starting point for many future researches. We can only conceive of apparent ones. As for example, the model can be extended to include limited capacity of the buses and the behaviour of the passengers under congestion (SUE). Another line of research is to satisfy in-day and day-to-day demand by taking into account dynamic frequency assignment. Finally, instead of only optimizing line frequencies, the decision of adding new or discontinuing existing lines can be also formulated and added to the model.

\section{References}

[1] Guihaire, V. and Hao J.-K., Transit network design and scheduling: a global review. Transportation Research Part A, 42(10), pp. 1-2, 2008.

[2] International Association of Public Transport (UITP). 10 $0^{\text {th }}$ Training Programme Report for Public Transport Managers: Corporate Management and Customer Relationships UITP Web Site, Santiago de Chile, 2012.

[3] Steg, L. and Gifford, R., Sustainable transportation and quality of life. Journal of Transport Geography, 13(1), pp. 59-69, 2005.

[4] Rusko, M. and Kotovicová, J., Environmental sustainability of transport. Research Papers Faculty of Materials Science and Technology Slovak University of Technology in Trnava, 26, pp. 55-62, 2009.

[5] Constantin, I. and Florian, M., Optimizing frequencies in a transit network: a nonlinear bi-level programming approach. Trans. Opl. Res., 2(2), pp. 149-164, 1995.

[6] Kurachi, F., Bell, M. G.H. and Schmöcker, J. D., Capacity constrained transit assignment with common lines. Journal of Mathematical Modelling and Algorithms, 2(4), pp. 309-327, 2003.

[7] Cepeda, M., Cominetti, R. and Florian, M., A frequency-based assignment model for congested transit networks with strict capacity constraints: characterization and computation of equilibria. Transportation Research Part B, 40, pp. 437-459, 2006. 
[8] Lam, W. H. K. and Ziliaskopoulos, A. K., A capacity restraint transit assignment with elastic line frequency. Transportation Research Part B, 36, pp. 919-938, 2002.

[9] Hamdouch, Y. and Lawphongpanich, S., Schedule-based transit assignment model with travel strategies and capacity constraints. Transportation Research Part B, 42, pp. 663-684, 2008.

[10] Cortés, C. E., Díaz, S. J. and Tirachini, A., Integrating short turning and deadheading in the optimization of transit services. Transportation Research Part A, 45, pp. 419-434, 2011.

[11] Poon, M. H., Wong, S. C. and Tong, C. O., A dynamic schedule-based model for congested transit Networks. Transportation Research Part B, 38, pp. 343-368, 2004.

[12] Xu, J., Liu, H. and Teng, J., A vehicle scheduling model and efficient algorithm for single bus line. Workshop on Power Electronics and Intelligent Transportation System, IEEE Computer Society, pp. 458-461, 2008.

[13] Guihaire, V. and Hao, J. K., Transit network timetabling and vehicle assignment for regulating authorities. Computers and Industrial Engineering, 59, pp. 16-23, 2010.

[14] Nielsen, O. A. and Frederiksen, R. D., Optimisation of timetable-based, stochastic transit assignment models based on MSA. Ann. Oper. Res., 144(1), pp. 263-285, 2006.

[15] Tong, C. O. and Wong, S. C., A stochastic transit assignment model using a dynamic schedule-based network. Transportation Research Part B, 33, pp. 107-121, 1999.

[16] Teklu, F., A stochastic process approach for frequency-based transit assignment with strict capacity constraints. Netw. Spat. Econ., 8, pp. 225240, 2008.

[17] Puchalsky, C. M., Use of bilevel optimization techniques for the problem of transit frequency determination: new formulation and solution techniques. Doctor of Philosophy Dissertation of Pennsylvania University, 2007.

[18] Sun, L. and Gao, Z. Y., An equilibrium model for urban transit assignment based on game theory. European Journal of Operational Research, 181, pp. 305-314, 2007.

[19] Yoo, G. S., Kim, D. K. and Chon, K. S., Frequency design in urban transit networks with variable demand: model and algorithm., KSCE Journal of Civil Engineering, 14, pp. 403-411, 2010.

[20] Soria Alves, D. A., Dantas, A., Yamamoto, K. and Yamashita, Y., Neural gee-spatial model for urban and transportation sustainability - system's diagnosis. WIT Press (Sustainabilty City II), pp. 1-2, 2002.

[21] Simões, A. M., Coelho, C. M., Silva, C. M. and Farias T. L., Analysis of the environmental impact of urban buses: application to a case study in Lisbon. WIT Press (Urban Transport VIII), pp. 1-2, 2002.

[22] Wadhwa, L. C., Sustainable transportation: the key to sustainable cities. WIT Press (The Sustainable city), pp. 1-2, 2000. 
[23] Latini, G., Cocci Grifoni, R. and Passerini, G., Sustainability of transportation systems: air pollution scenarios. WIT Press (Transactions on Ecology and the Environment), 67, pp. 1-2, 2003.

[24] Lam, W. H. K. and Bell, M. G. H., Advanced Modelling for Transit Operations and Service Planning. Elsevier Science Ltd.: Oxford, pp. 165170, 2003.

[25] Deb, K., Pratap, A., Agarwal, S. and Meyarivan, T., A fast and elitist multiobjective genetic algorithm: NSGA-II. IEEE Transactions on Evolutionary Computation, 6(2), pp. 182-197, 2002.

[26] Lents, J., Unal, A., Mangir N., Osses, M., Tolvett, S. and Yunusoglu, O., Study of the emissions from diesel vehicles operating in Istanbul, Turkey: Technical Report. International Sustainable Research Center, pp. 4-5, 2007. 